Selecting the Best Approach to Teach 3D Modeling to Technical College Engineering Students

Dr. Farzin Heidari, Texas A\&M University, Kingsville 


\title{
Selecting the Best Approach to Teach 3D Modelling to Technical College Engineering Students
}

\begin{abstract}
The paper will provide a step-by-step methodology to enable an instructor dealing with 3D modelling to optimally guide his/her students through an understandable 3D modelling approach which will not only enhance their knowledge about the CAD program usage but also enable students to achieve their desired result in comparatively less time. Grid method, origin reference method and planes reference methods to create sketches and 3D models will be discussed.

The advantages of planes reference method in comparison to origin reference method will be presented. The information provided by this paper will serve as a source to improve the quality of technical college instructors teaching 3D modelling to engineering students.
\end{abstract}

\section{Introduction}

There are currently many CAD design packages available in the market. There are four common standard design tools to display CAD's overall functionalities. They are respectively:

\begin{tabular}{ll} 
- & SolidWorks \\
- & Pro/Engineer \\
- & CATIA \\
\hline & Autodesk Inventor
\end{tabular}

Among all the above mentioned tools, SolidWorks is the most widely used software in industry and also it is taught to students in most of the educational institutions. Hence it is selected to be used as training tool in this paper.

SolidWorks is a 3D parameterized design tool, focusing on Para-solid inclined solid modelling environment. ${ }^{1}$ Drawings in SolidWorks can be worked out from previous assemblies or assembly portions. View generations are automatic and acquired from the solid model itself. Tolerances, notes and dimensional feedbacks, as per the requirement, can be added later on. Modules can be prepared on all standard orientations and layouts like ANSI, ISO, JIS.

SolidWorks can also create fresh solid models from scratch using advanced geometry tools. Some terms associated with a typical SolidWorks working environment are as follows:

Parameters: Constraint values determining overall geometrical shape of the design. Classified into Geometric (concentricity, tangency, parallelism etc.) and Numeric (length, diameter etc.), these parameters establish relations between separate assembly portions to give an overview of the design intent. ${ }^{1,2}$

Design Intent: Physical significance of the design part. Established through constraint equations (parameters), design intent is utilized by the designer for adaptation of the specific portion in regards to modifications done to the model as a whole. 
Features: Holes, slots etc. 2D or 3D sketch-based design inclusions specifically made for further additions into the model. They are the building blocks, constructing and shaping a particular part of the design.

Mates: In SolidWorks, Mates (Assembly Mates) are analogous to mathematical relations in a sketch. Such relations define equivalency amongst different components of the assembly, allowing easy construction of the same. ${ }^{3}$

Mathematical relations define the model structure in SolidWorks and they are responsible for the modifications incorporated into the design later on, making the absolute parametric CAD design tool.

- Designing starts as simply as a 2D sketch. Such a 2D sketch includes basic geometric components like arcs, straight lines, conics etc.

- Dimensions are incorporated into the sketch to provide size and a 3D model shape to the design. Locations are added to the geometry components. Such dimensions are independently controlled.

- Relations within variables (parameters) are allocated which introduce advance constraint functions like concentricity, parallelism, tangency etc. Dimensions within the design can also be controlled or altered with the help of parameters in or out of the underlying sketch.

- Once the different parts are created, assembly mates are included to conjugate the whole solid model. Apart from assembly, there are also other advanced mating tools like gear mating to reproduce accurate rotational movements in gear trains.

- The idea, once conceived into the solid model drawings, can be shared for feedbacks. Dimension and tolerance can be adjusted with the revised and provided inputs.

Following are the three common methods learned by students to create a sketch to develop a 3D model:

\section{Grid Method:}

A grid is defined as a framework of adjacent squares formed by horizontal and vertical lines that resembles a graph sheet. Every grid has a specific Origin point $(0,0)$ and every other point on the grid is defined by the coordinates taking the Origin as the major reference. The grid helps as a map to the students to plot the points of the sketch to be drawn taking the coordinates as reference.

Usually the Origin point $(0,0)$ of a grid can be any point in the grid. Depending on the position where the origin is fixed, the remaining points on the grid need to be labelled. Few designers consider the lower left corner of the grid as the origin and label the rest of the grid as the first quadrant where all the points are positive, while some other designers consider the centre point as the origin and label the rest of the grid into four different quadrants similar to a coordinate system. The value of each unit of a grid depends on the sketch and the scale to which the sketch needs to be reproduced. ${ }^{2,3}$ 
Figure 1 is an example of a grid with its lower left corner as the Origin $(0,0)$.

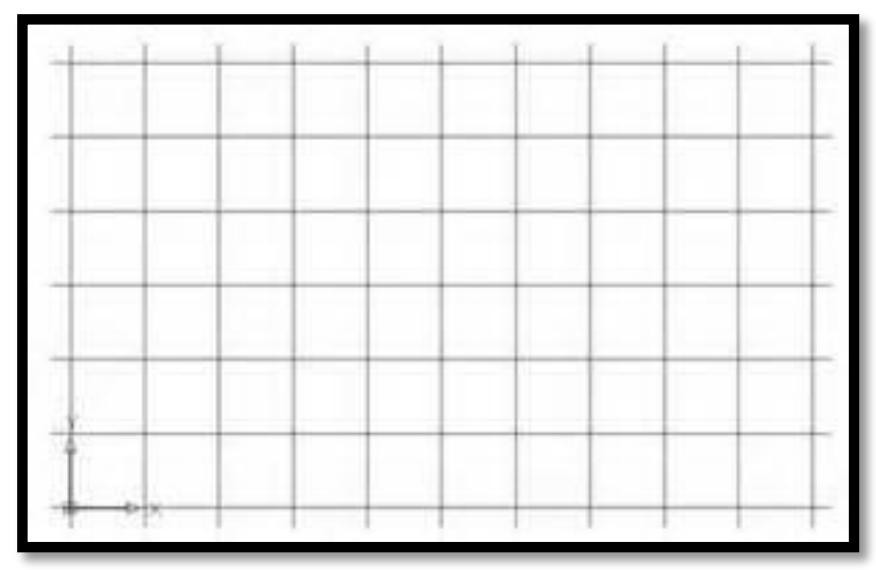

Fig.1. Basic Grid Structure

How to draw a sketch using grid:

For any designer to draw a sketch on a grid, defining the position of the Origin $(0,0)$ and labelling the rest of the grid is the first step. Depending on the size of the sketch, the value of each unit of the grid is defined and all the coordinates on the grid are labelled accordingly. From the position of the origin, all the points on to the right are labelled as positive and those on the left are labelled as negative. Similarly, the points above the origin are labelled as positive and those below are labelled as negative. The points are identified as ordered pairs with their unit values within the parenthesis, like $(\mathrm{X}, \mathrm{Y})$. The first number $(\mathrm{X})$ of the ordered pair defines the location of the point on the grid in horizontal direction and the second number $(\mathrm{Y})$ defines the location of the point in vertical direction, may it be positive, negative or zero. For example, the coordinate $(5,3)$ lies 5 units away from the origin towards its right and 3 units away from the origin in the upward direction.

It is not mandatory to start every sketch considering the origin $(0,0)$ as its starting point. A sketch can be started anywhere on the grid and can be continued in any direction as per the ease and the practice of the designer using the ordered pairs that are labelled on the grid.

The grid system approach of sketching any drawing is spoken as something old fashioned and it is actually more time consuming when compared to regular method of drawing. Though there are cases where using a grid are the only method of approach to develop a sketch, it is not a preferred method when it comes to drawing 2D sketch on a 3D CAD system.

\section{Origin Reference Method:}

This is the general method of approach widely taught to students in most educational institutions in teaching various $3 \mathrm{D}$ CAD systems. It can be considered as routinely followed method in today's educational system for instruction to beginners. One of the three common planes, Front Plane, Top Plane or Right Plane is selected. The selected plane is adjusted to the normal view of the designer, then the origin of the selected plane is used to start the skatch. 
In this method, Origin $(0,0)$ on the selected plane, which is located in the centre of the plane is considered as the major reference to create the sketch of any cross section to be developed. For example, a circle is drawn using the origin as its centre while a rectangle is drawn using the origin as its lower left corner. Except the circle, almost all the other sketch profiles begin at the origin and are developed towards right in the positive direction into the first quadrant, irrespective of the shape of the part. In this method, the extrusion of any part is unidirectional most of the times. ${ }^{4}$

Figure 2 illustrates an example of one such sketch that is being created using Origin reference method.

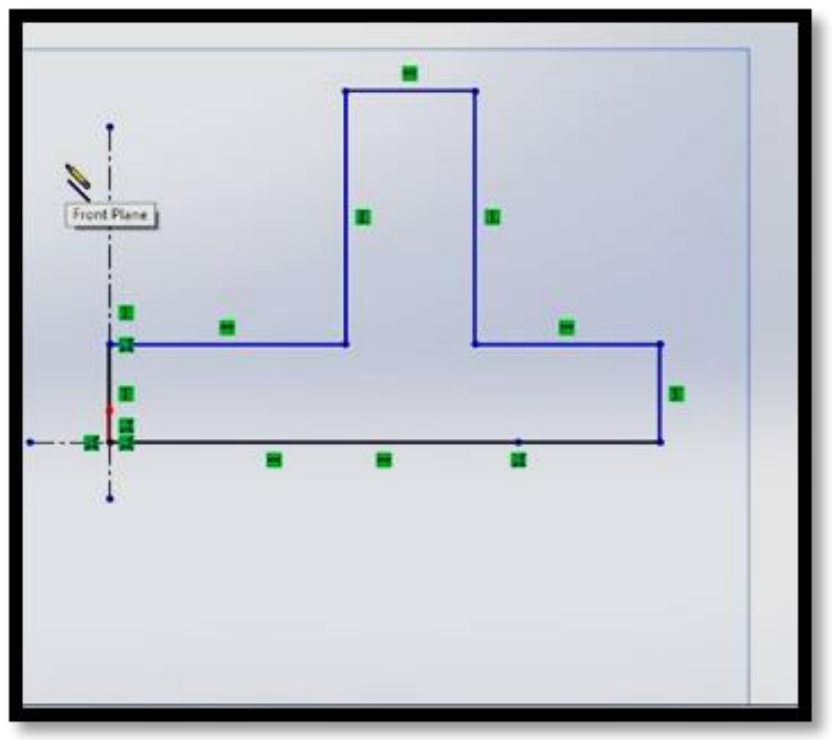

Fig.2. Typical Origin Reference Method Sketch

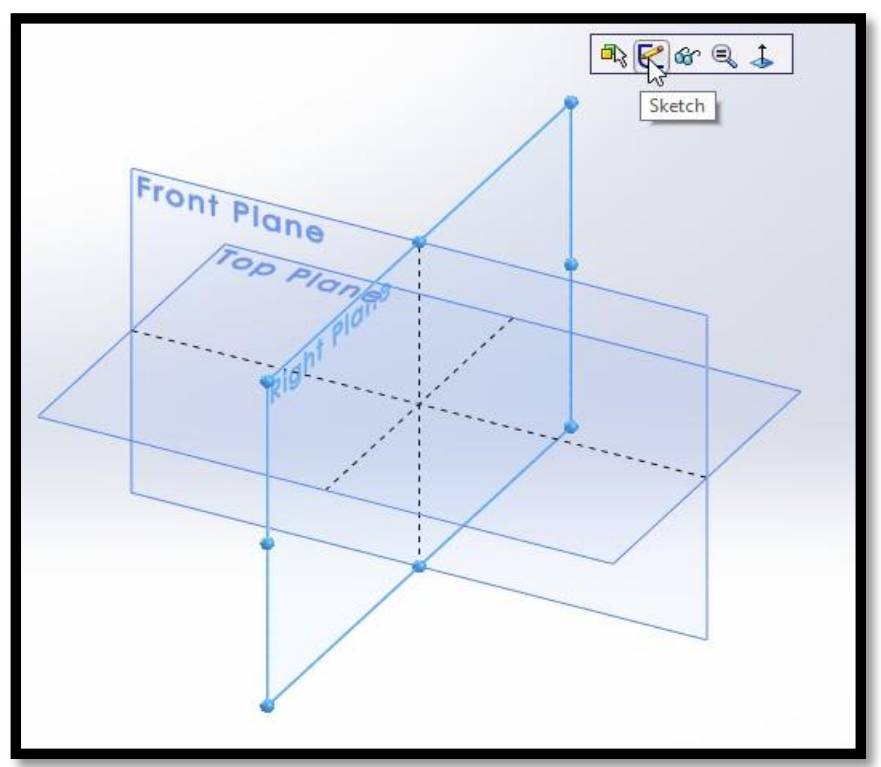

Fig. 3 Plane Reference Method 
Planes Reference Method:

In this method, along with the coordinate axes, the datum planes are also given the equal importance in creating and developing a sketch. According to this method, all the individual components that are part of the final assembly are built with respect to the orientation of the final assembly of a model which a designer assumes. The three planes are activated and the centre of the plane that best describe the parameter of the part is used as the centre of the part.

Figure 4 illustrates an example of one such sketch that is being created using Planes reference method.

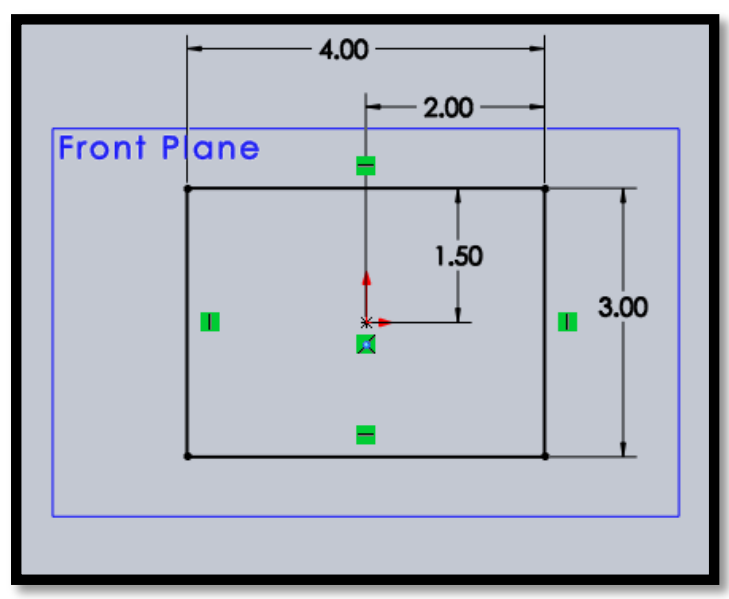

Fig.4. Typical Planes Reference Method Sketch

After parts are generated using the above methods the Mate command is used to orient and position the components in the assembly. An assembly combines two or more parts. In assembly, parts are referred to as components. The components are inserted into the assembly file and mated together as they related to the physical limitation of the design. Mates provide the ability to create geometric relationship between assembly components.

There are two different types of Mate commands, standard Mates and advanced Mates. Standard Mates are Coincident Mate, Perpendicular Mate, Tangent Mate and Concentric Mate. Standard Mates are used in the parts created by grid and origin reference method. ${ }^{5,6}$ Advanced Mates commands are Symmetric Mate, Width Mate and Path Mate. The Symmetric Mate is readily adapted to the plane reference method, it positions two selected entities to be symmetric about a plane or planar face. ${ }^{5}$ This advanced feather which provides fast and easy assembly between components will not work in gird or origin reference method because no planar relationship exists between part. ${ }^{5}$

Advantages of Planes reference method over the Origin reference method:

- Less time is taken in executing the project, once the method gets adapted.

- Enables easy editing of the components as well as the assemblies at any stage of modelling. 
- Helps in following a well-organized manner of practicing 3D modelling using CAD systems.

- Helps students realize the importance of understanding the actual application of the model, before starting working on it.

- Following a standard method of approach avoids delays in executing the project. For example, if each individual follow their own method of approach in creating a 3D model which is a part of a project, then even a short period of absence of that particular individual in that project sometimes leads to unforeseen and unpredictable delays in delivering the projects. As no other person can understand the strategy that one person follows, it results in holding on to that part of work until the delegated person is back at work. This is one of the major problems which can be often experienced in current industry.

- Hence, standardization of methods and processes can help in avoiding many issues in different stages of any project.

Table1. Comparison between two reference methods

\begin{tabular}{|c|c|}
\hline Origin Reference Method & Planes Reference Method \\
\hline $\begin{array}{l}\text { - Origin is considered as the major } \\
\text { reference to create a sketch. } \\
\text { - Most of the times, just the } \\
\text { orientation of an individual part is } \\
\text { taken into consideration. } \\
\text { - For mating the parts in an } \\
\text { assembly, only the surfaces of the } \\
\text { adjacent parts are considered. } \\
\text { - Less time taking comparatively. } \\
\text { - A very easily understandable } \\
\text { method of approach for beginners } \\
\text { though not suggestible, if } \\
\text { compared to real-time practice. }\end{array}$ & $\begin{array}{l}\text { - Planes are considered as major reference } \\
\text { to create a sketch. } \\
\text { - Orientation of the whole model is } \\
\text { assumed as the first step and then } \\
\text { individual part orientation is planned. } \\
\text { - Datum planes are used in all possible } \\
\text { ways to define the mates in the } \\
\text { assembly. } \\
\text { - Takes more time in planning but easily } \\
\text { executable. } \\
\text { - Though not easy in the initial stage of } \\
\text { learning to adapt to this method, it turns } \\
\text { out to be very helpful for a designer } \\
\text { when they start a profession as 3D } \\
\text { modeller. }\end{array}$ \\
\hline
\end{tabular}




\section{Conclusion:}

Current educational curriculums related to 3D modelling do not provide adequate information to teach real-time practices of mating programs. Most students follow the trend of accepting the Origin $(0,0)$ as the starting point of the sketch and the whole design is built on right-hand side of the first quadrant. The physical shape of the object, whether it should be symmetric or nonsymmetric, is not considered. Even if the students realise the part to be symmetric they still prefer to develop the whole design on the first quadrant. Using the symmetry features through applying planes reference method improves the quality of 3D modelling for engineering students.

\section{References}

1. Ambler, S.W. (2003, December). User Interface Design: Tips and Techniques. Retrieved from http://www.ambysoft.com

2. Bertoline, G., Wiebe, E. N. and Miller, C. L. (1998) Fundamentals of Graphics Communication $\left(2^{\text {nd }}\right.$ ed.) The Mc-Graw Hill Companies, Inc.

3. Kim, G. J. (1997). Case-base design for assembly. Computer-Aided Design, 29(70, 497-506.

4. Narayan, L. K. (2008). Computer Aided Design and Manufacturing. New Dehli, India: Prentice Hall of India.

5. Planchard, D., Planchard, M. (2013). A Step-by-Step project Based Approach Utilizing 3D Solid Modeling. Mission, KS: Schroff Development Corporation.

6. Reyes, A., (2015). Beginner's Guige to SolidWorks. Mission, KS: SDC Publication. 